\title{
A novel miniature transposon-like element discovered in the coding sequence of a gene that encodes for 5 - formyltetrahydrofolate in wheat
}

\author{
Katherine Domb ${ }^{1+}$, Danielle Keidar-Friedman ${ }^{2+}$ and Khalil Kashkush ${ }^{2^{*}}$ (D)
}

\begin{abstract}
Background: Transposable elements (TEs) comprise over $80 \%$ of the wheat genome and usually possess unique features for specific super-families and families. However, the role of TEs in wheat evolution and reshaping the wheat genome remains largely unclear.

Results: In this study, we discovered a miniature (307 bp in length) TE-like sequence in exon 6 of a gene that encodes for 5-formyltetrahydrofolate, in two accessions of wild emmer wheat (T. turgidum ssp. dicoccoides) and has interfered with the gene translation by creating a shorter reading frame as a result of a stop codon. The sequence that was termed Mariam, does not show any structural similarity to known TEs. It does not possess terminal inverted repeats (TIRs) that would allow us to assign this element to one of the TIR DNA super-families, and it does not possess characteristic features of SINE, such as a Pol-III promotor or a poly-A tail. In-silico analysis of five publicly available genome drafts of Triticum and Aegilops species revealed that Mariam element appears in a very low copy number (1-3 insertions) in diploid wheat species and $\sim 12$ insertions in tetraploid and hexaploidy wheat species. In addition, Mariam element was found to be unique to wheat, as it was not found in other plant genomes. The dynamic nature of Mariam in the wheat genome was assessed by site-specific PCR analysis and revealed that it retained activity in wild emmer populations in a population-specific manner.
\end{abstract}

Conclusions: This study provides additional insight into the evolutionary impact of TEs in wheat.

Keywords: Miniature transposable elements, Triticum, Aegilops, 5-formyltetrahydrofolate, Genome evolution, Polyploidy, Wheat

\section{Background}

Transposable elements (TEs) are DNA segments that have the ability to proliferate within their host, as such, they can make up large fraction of eukaryotic genomes [1]. The wheat genome harbor thousands of known TE families that occupy $\sim 80 \%$ of the genome, while LTR retrotransposons are the most abundant [2-5]. TEs are divided into Class I elements (retrotransposons) and Class II elements (DNA transposons), which are further divided into super-families and families [6].

\footnotetext{
* Correspondence: kashkush@bgu.ac.il

${ }^{\dagger}$ Katherine Domb and Danielle Keidar-Friedman contributed equally to this work.

${ }^{2}$ Department of Life Sciences, Ben-Gurion University, 84105 Beer-Sheva, Israel Full list of author information is available at the end of the article
}

TEs can be stimulated by various biotic or abiotic stresses such as heat shock, wounding or bacterial infection [7-9], as well as genomic stresses such as hybridization and polyploidization [10]. Activity of TEs in the genome can alter its structure both genetically and epigenetically [11-14]. Insertions of TEs into coding regions (exons) can cause disruption of function and generation of mutant phenotypes [15], while insertions into introns can interfere with transcript editing or lead to intron retention [16-18]. Insertions of TEs in close proximity to genes can affect their expression, for example by interfering with promoter activity $[15,16]$. One other known consequence of TEs amplification is expansion of

(c) The Author(s). 2019 Open Access This article is distributed under the terms of the Creative Commons Attribution 4.0 International License (http://creativecommons.org/licenses/by/4.0/), which permits unrestricted use, distribution, and 
the host genome size $[14,19]$. All these effects have a key role in allelic and phenotypic diversity of plants [14].

Wheat (Triticum-Aegilops group) had originated $\sim 4$ million years ago following the divergence of three diploid species from a common ancestor. The tetraploid T. turgidum ssp. dicoccoides (wild emmer, $\mathrm{AB}$ genome) was generated by an allopolyploidization event that included hybridization of Triticum urartu (donor of A genome) and an unknown Aegilops species (of section sitopsis, donor of B genome). The primary domestication of wild emmer and the following evolution of hulled domesticated emmer wheat (T. turgidum ssp. dicoccun) induced the selection of free-threshing durum (T. turgidum ssp. durum, AB genome). The hexaploid Triticum aestivum (Bread wheat, ABD genome) was generated $~ 10,000$ years ago by a second major polyploidization event that involved the hybridization between domesticated emmer wheat (T. turgidum ssp. dicoccun) and Aegilops tauschii (donor of D genome) $[4,20,21]$. Wild emmer is found in nature as a wild species and since its rediscovery by Aharon Aharonson in 1913 [22], this species has been extensively studied as a potential donor of beneficial traits to domesticated wheat [22-26]. Wild emmer, an annual, predominantly self-pollinating species, is distributed in a patchy manner throughout the Middle East in diverse environmental conditions that vary in average annual temperature, altitude, soil type, and other conditions. In Israel, there are over 20 populations (isolated or semi-isolated) of wild emmer that can be found in regions between Mt. Hermon in the north and Mt. Amasa (Judea desert) in the south [27, 28]. We recently showed that Transposable elements can proliferate in a population-specific manner in wild emmer wheat, thus creating allelic variation [29].

In this study, we present the discovery of a wheatunique miniature TE-like sequence termed Mariam in two accessions of a marginal population of wild emmer wheat. The availability of 5 recently updated wheat genome drafts; T. turgidum ssp. dicoccoides, T. turgidum ssp. durum, T. aestivum, Ae. Tauschii and T. urartu, facilitated the computer-assisted analysis of Mariam content and dynamics in these wheat species. In addition, the impact of the novel Mariam insertion in a gene that encodes for 5 -formyltetrahydrofolate was assessed, as well as the dynamics of Mariam in wild emmer wheat populations. To this end, the evolutionary impact of Mariam insertions is discussed.

\section{Results}

A novel TE-like DNA fragment discovered in the coding sequence of a gene that encodes for 5 formyltetrahydrofolate in Mt. Hermon population of wild emmer wheat

As part of a study [29] that aimed to identify and characterize polymorphic insertions of miniature inverted- repeat transposable elements (MITEs) in five wild emmer wheat populations, we have discovered a short DNA insertion (307 bp in length) in exon 6 of a gene that encodes 5formyltetrahydrofolate (TRIDC2AG023940, EnsemblPlants) in two accessions of Mt. Hermon population wild emmer populations (Fig. 1 top). Genome-specific primers were designed from intron 5 upstream to a MITE insertion, termed Fortuna, and from exon 6 of the gene (Fig. 1, Additional file 1: Table S1). The expected size of the full site was $543 \mathrm{bp}$. The site-specific PCR experiment showed that Fortuna element is present in all accessions of these five populations (Mt. Hermon, Amiad, Tabgha, Jaba and Mt. Amasa), yet in two accessions of the Mt. Hermon population, a higher band of $850 \mathrm{bp}$ was amplified instead of the expected full or empty site (Fig. 1 bottom).

Sequence analysis of the $307 \mathrm{bp}$ insertion (Fig. 2) revealed that this sequence did not hit any known sequence from the database including genomic and transcriptomic databases (https://plants.ensembl.org/Triticum_aestivum/ Info/Annotation/ [30], http://plants.ensembl.org/Triticum dicoccoides/Info/Annotation/ [4]), and repeat databases such as ITMI [31], the Triticeae repeats database (http:// botserv2.uzh.ch/kelldata/trep-db/index.html), and GIRI database [32] (http://www.girinst.org/censor/index.php) that yielded no hits to annotated transposable elements. Interestingly, the 307 bp sequence was flanked by a 9 bp sequence (CCAAGAACT) at both ends resembling a targetsite duplication (TSD) that can be generated as a result of transposable elements insertions (Fig. 2). The fact that the $9 \mathrm{bp}$ site was found in only one copy in the gene lacking the new insertion indicates the $307 \mathrm{bp}$ sequence was integrated in a TE-like manner, thus creating TSD. Furthermore, the $307 \mathrm{bp}$ insertion does not possess terminalinverted repeat sequences (TIRs) that would allow us to assign this sequence to one of the TIR DNA superfamilies. In addition, it does not possess characteristic features of SINEs such as a Pol-III promotor or a poly-A tail.

To test whether other copies of the TE-like insertion appear in Triticum-Aegilops genomes, MAK software was used to retrieve TE-like insertion sequences from the recently available genome drafts. We have found one insertion in T. urartu (AA genome), 3 insertions in Ae. tauschii (DD genome), 12 insertions (8 in subgenome A and 4 in subgenome B) in T. turgidum ssp. dicoccoides (wild emmer, AABB genome), 6 insertions (4 in subgenome A and 2 in subgenome B) in T. turgidum ssp. durum (durum, AABB genome) and 11 insertions (3 in subgenome A, 4 in subgenome $\mathrm{B}$ and 4 in subgenome $\mathrm{D}$ ) in $T$. aestivum (bread wheat, AABBDD genome). Sequences of all retrieved insertions and their molecular charactrization, including sub-genome and chromosmal locations can be found in Additional file 1: Table S1. Note that this insertion was found to be unique to Triticum-Aegilops group as it was not found in other plant genomes. 


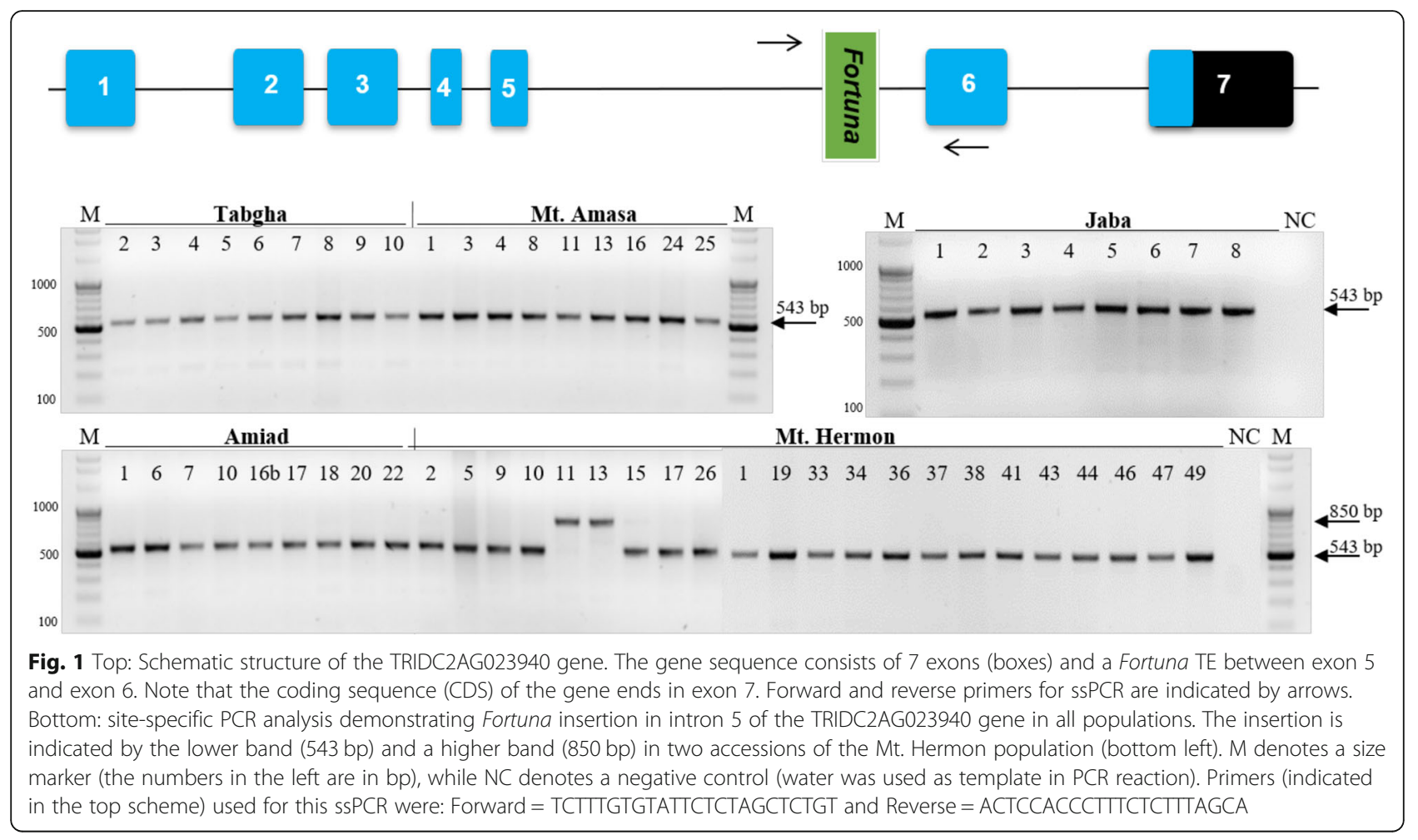

A sequence logo was created from the flanking sequences of the 33 full-length $307 \mathrm{bp}$ insertions retrieved from the 5 genome drafts using WebLogo software [33] (http://weblogo.threeplusone.com/, Additional file 4: Figure S1). While 16 of the 33 insertions showed clear TSD, the remaining 17 insertions did not show notable TSD (Additional file 1: Table S1). The sequence logo demonstrated a certain sequence preference for specific nucleotides at positions 4 and 9, however the 9-bp duplicated sequences were not highly conserved. The lack of detectable TSDs for 17 insertions can be explained by mutations /rearrangements around the insertions that might have disrupted the formerly identical sequences flanking each insertion. Note that over 50\% (9 out of 17) of the insertions lacking TSD are located in genome BB, which is known to be very dynamic $[20,21]$. To this end, the fact that clear TSDs of conserved length were detected in $\sim 50 \%$ of the insertions supports the hypothesis of a transposon-like behaviour, therefore we named this TIR-less, minature element "Mariam". Note that multiple sequence alignment analysis revealed high sequence conservation (over 90\%) of the 33 full-length Mariam elements retreived from the five genome drafts. The high sequence conservation of Mariam among diploid and polyploid species might strongly indicate a recent prolifiration of this element in wheat.
Insertional polymorphism of Mariam in Triticum-Aegilops group and within wild emmer populations

As mentioned above, the existence of Mariam elements in T. urartu and Ae. tauschii, and in the three sub-genomes of bread wheat suggests this element was probably present in all diploid progenitors of the wild emmer and bread wheat and was amplified in the polyploid species. Of the 7 Mariam insertions found in A and B sub-genomes of T. aestivum, 6 were common (monomorphic insertions) to wild emmer. In addition, all 3 Mariam insertions found in Ae. tauschii were common to the D sub-genome of T. aestivum. Finally, the insertion found in T. urartu was common to wild emmer and to T. aestivum. The dynamics of Mariam elements in wild emmer wheat and in bread wheat accessions were assessed using site-specific PCR analysis on DNA isolated from 45 wild emmer accessions (collected from 5 different goegraphically isolated populations; Mt. Hermon, Amiad, Tabgha, Jaba and Mt. Amasa - 9 accessions from each population) and 8 bread wheat accessions (see Additional file 2: Table S2). Overall, primers were designed from flanking sequences of 10 Mariam insertions mapped to A and B sub-genomes of wild emmer and/or bread wheat (Additional file 3: Table S3).

The results of the PCR analysis demonstrated high insertional polymorphism levels of Mariam based on presence (full site) vs. absence (empty site) among wild 


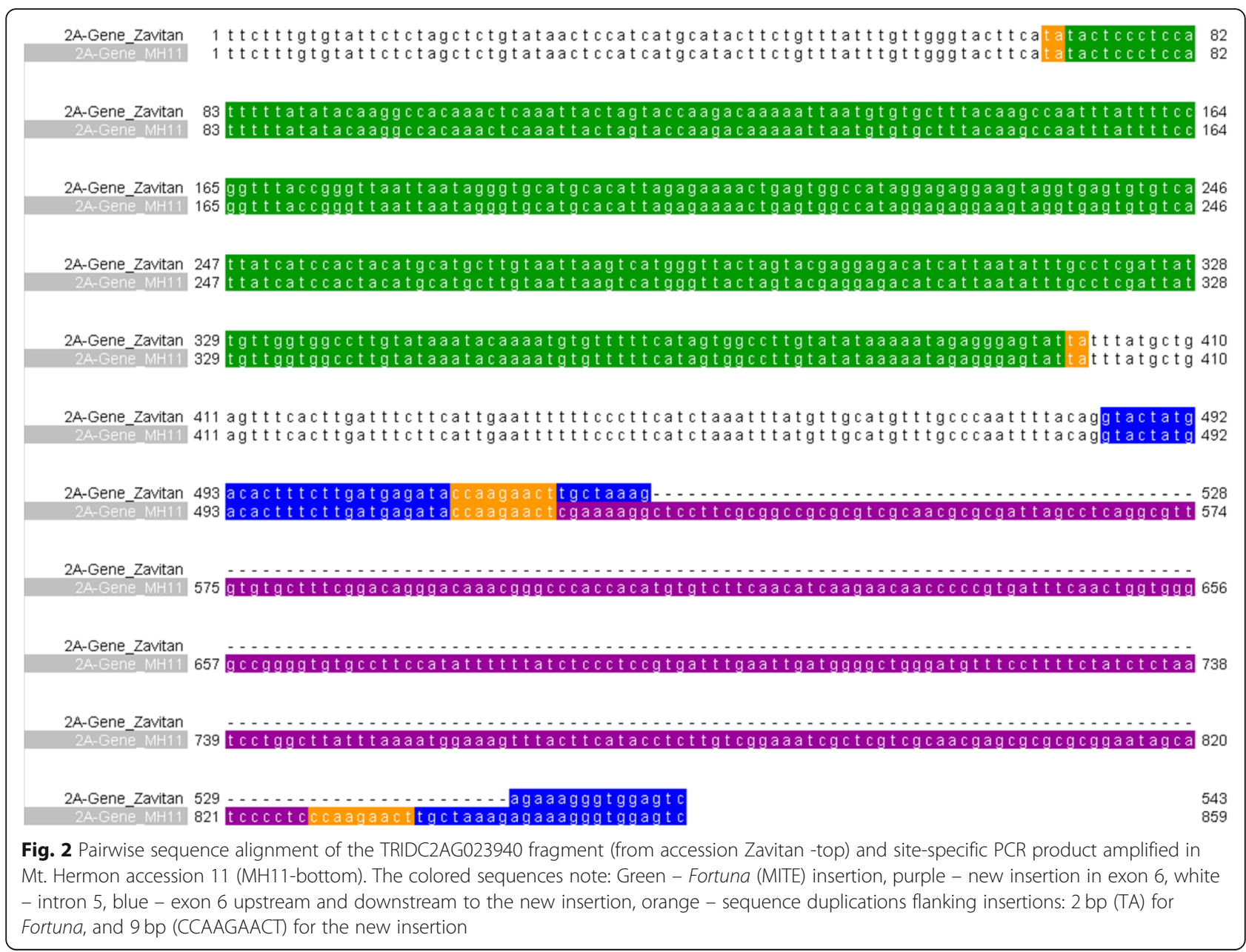

emmer wheat accessions. Only one of the examined insertions, A5-6 (Table 1), was present in all accessions of wild emmer and bread wheats (monomorphic insertion), while 6 insertions (A4-4 (Fig. 3), A6-2, A7-5, B3-4, B7-4, B7-6 (Additional file 4: Figure S2) were polymorphic in wild emmer wheat accessions in a population-specific manner and were not detected in the bread wheat accessions (Table 1). One insertion (B1-4, Table 1) was absent from all wild emmer wheat accessions but was present in all bread wheat accessions. In two additional cases (A4-1 (Additional file 4: Figure S2f), B14. (Additional file 4: Figure S2g)), insertions were polymorphic in both wild emmer wheat accessions and in bread wheat accessions. A4-1 insertion exists in only one of eight bread wheat accessions, while B1-4 insertion exists in seven of eight accessions.

The insertional pattern of Mariam (according to the results of the ssPCR analysis) was used to build a phylogenetic tree generated by hierarchical agglomerative clustering (see Fig. 4). The phylogenetic tree showed significant ( $p$-value $<0.05)$ separation between Tabgha population (except for T3 accession) and other populations, which correlates with our previous finding according to Minos (a MITE family) insertional pattern that was assessed Transposon Display assay [29]. All T. aestivum accessions were clustered together, as well as Mt. Amasa and Amiad that were generally clustered by their population, except for two of their accessions. Jaba and Hermon populations showed high variability among accessions within population. These findings might indicate the population-specific dynamics of Mariam in wild emmer wheat and in bread wheat species.

\section{Mariam insertions are associated with wheat genes}

Sequence annotation of Mariam insertion sites revealed that 11 of the 33 Mariam insertions were located adjacent to other TE elements including class I and class II elements (Additional file 1: Table S1), while 12 insertions were located into or adjacent (up to $500 \mathrm{bp}$ downstream or upstream) to genes, such as formyltetrahydrofolate cyclo-ligase, sucrosephosphatase 3, soleucyl-tRNA synthetase, Serine/ threonine-protein kinase, xyloglucanase and others. This is in addition to the previously described 
Table 1 Insertional polymorphism of Mariam in wild emmer wheat and in bread wheat accessions based on ssPCR analysis

\begin{tabular}{|c|c|c|c|c|c|c|}
\hline \multirow[t]{3}{*}{ Locus $^{a}$} & \multicolumn{6}{|c|}{$\begin{array}{l}\text { Presence (full site)/ absence (empty site) of Mariam in five wild emmer populations ( } 9 \text { accessions from each population) and in } 8 \text { bread } \\
\text { wheat accessions }\end{array}$} \\
\hline & \multicolumn{5}{|c|}{ Wild emmer populations (T. turgidum ssp. dicoccoides) } & \multirow{2}{*}{$\begin{array}{l}\text { Bread wheat } \\
\text { (T. aestivum) }\end{array}$} \\
\hline & Mt. Hermon & Amiad & Tabgha & Jaba & Mt. Amasa & \\
\hline $\mathrm{A} 2-\mathrm{MH}$ & 1 & 0 & 0 & 0 & 0 & 0 \\
\hline A4-1 & 0 & 1 & 1 & 0 & 0 & 1 \\
\hline A4-4 & 1 & 1 & 1 & 1 & 1 & 0 \\
\hline A5-6 & 2 & 2 & 2 & 2 & 2 & 2 \\
\hline A6-2 & 0 & 1 & 1 & 0 & 0 & 0 \\
\hline A7-5 & 0 & 1 & 1 & 0 & 1 & 0 \\
\hline B1-2 & 0 & 0 & 0 & 0 & 0 & 2 \\
\hline B1-4 & 1 & 1 & 2 & 1 & 1 & 1 \\
\hline B3-4 & 0 & 1 & 1 & 0 & 0 & 0 \\
\hline B7-4 & 0 & 1 & 1 & 1 & 0 & 0 \\
\hline B7-6 & 0 & 1 & 1 & 0 & 0 & 0 \\
\hline
\end{tabular}

${ }^{a}$ The chromosome where the insertion was found, and an additional identifier of each insertion site

${ }^{\mathrm{b}}$ The presence/absence of a TE insertion in examined accessions of a given population (for $T$. dicoccoides) or a given species (T. aestivum): 0 - empty site in all accessions, 1 - full site in some accessions, 2 - full site in all accessions. See Figs. 1 and 3 and Additional file 4

Mariam insertion (A2-MH) in exon 6 of the TRIDC2AG023940 gene encoding a predicted mitochondrial 5-formyltetrahydrofolate cyclo-ligase.

To test whether Mariam insertion in the gene coding sequence has any impact on the gene activity, we designed primers for RT-qPCR analysis from TRIDC2AG023940 gene and/or its homolog from the B genome. The relative expression level of each accession was normalized to the relative expression level of an arbitrary chosen accession (T1) to enable comparison between samples. The relative expression levels of TRIDC2AG023940 and/or its homolog from the B subgenome were found to vary within and between populations of wild emmer wheat (Fig. 5).

Amiad, Tabgha and Jaba populations demonstrated over 2-fold differences in expression levels of this gene between some accessions, while the variation within the Mt. Amasa population was less prominent. Mt. Hermon population demonstrated significantly lower expression level than Amiad Population (one-way ANOVA, $F=$ 4.3655, $p$-value $=0.0169$, Fig. 5). In addition, $\sim 2$-fold variation was seen within the different accessions in Mt. Hermon population, while the accessions that harbor Mariam insertion within the TRIDC2AG023940 gene showed the lowest expressions levels. For example, accession $\mathrm{H} 11$ shows reduction in the expression level of TRIDC2AG023940 gene vs. other accessions in Mt. Hermon population. These results might indicate the complex control of the expression of this gene, and do not allow validation or ruling out of the possible effect of this Mariam insertion on transcript quantity. However, in this case, the insertion was present in an exon and thus might either interfere with splicing or remain in the mature RNA. To test these possibilities, primers complementary to the predicted cDNA sequence (harbor Mariam insertion) were designed for RT-PCR analysis. The expression analysis was performed using cDNA of 9 accessions from the Mt. Hermon population as template. In all examined accessions where no insertion in exon 6 was detected (see Fig. 1), a single clear band corresponding to the expected $626 \mathrm{bp}$ size of the spliced product could be seen (Fig. 6). In the H13 accession where the insertion in exon 6 was present at the DNA level (Fig. 1), there was a single higher band, corresponding to the expected $942 \mathrm{bp}$ size of a spliced product with an insertion in exon 6 (Fig. 6). For validation, this higher band in $\mathrm{H} 13$ accession was extracted from the agarose gel, purified and sequenced, and found that this transcript is indeed harboring the full-length Mariam insertion. This experiment demonstrates that the insertion in exon 6 altered the mature mRNA sequence produced from the gene in a genome-specific manner.

According to the coding sequence (CDS) prediction for this gene in the EnsemblPlants database, CDS starts in exon 1 and ends in exon 7. Therefore, if the transcript is translated, the new insertion in exon 6 will interfere with translation. ORF analysis for cDNA with the new insertion predicted a stop codon at position 41 out of the 307 nucleotides of the new insertion, so that the protein product produced from the allele with the insertion in exon 6 would possess a shortened and altered Cterminus. A Blastp search of the translated cDNA of the TRIDC2AG023940 gene without the insertion (Fig. 7a) and with the insertion in exon 6 (Fig. 7b) in the NCBI database demonstrated that the insertion interferes with the C-terminal part of the cyclo-ligase domain. 


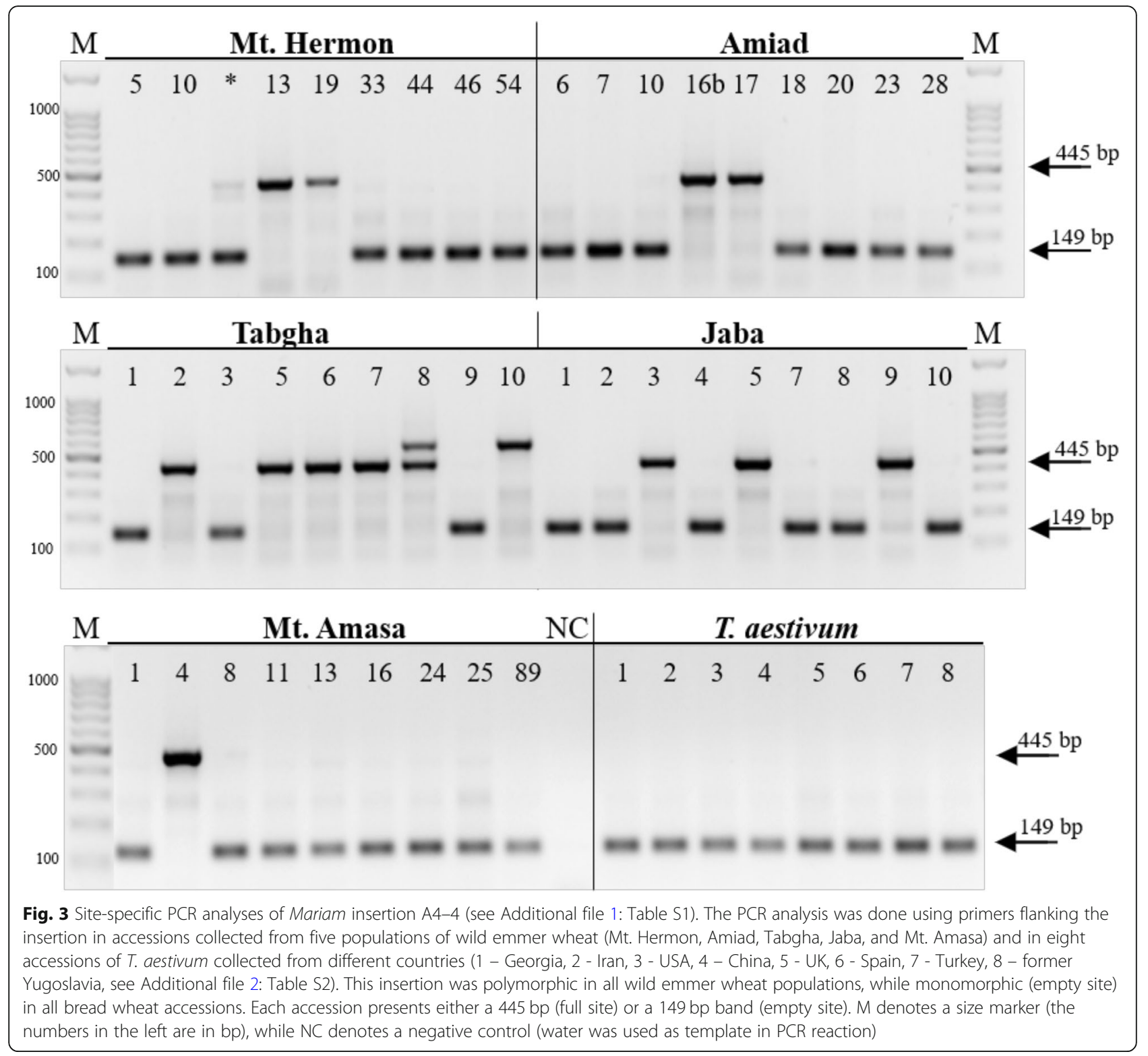

\section{Discussion}

In this study, we present a novel miniature TE-like sequence first discovered in wild emmer wheat. This sequence, initially found in two accessions of Mt. Hermon population of wild emmer wheat and termed Mariam, is a low-copy number repetitive sequence. This fragment was found in different wheat species in relatively small numbers, specifically between single insertions in diploid species such as T. urartu and in around 12 insertions in wild emmer and bread wheat. The first insertion of Mariam discovered, when sequenced, resembled a TE insertion in that it generated a TSD. However, no similar sequence was found in any database of repetitive sequences and TEs. In addition, the small size (307 bp) of Mariam indicates that it is non-autonomous and might be classified to miniature inverted-repeat transposable elements (MITEs).

The sequence of Mariam does not show any functional similarity to known TEs. It does not possess TIRs that would allow us to assign this element to one of the TIR DNA element super-families. Furthermore, it does not possess any characteristic features of SINEs, such as a Pol-III promotor or a poly-A tail. The only feature that Mariam element has in common with a known TE superfamily is the length of the sequence duplications flanking its intact insertions in $\sim 50 \%$ of cases. Target site duplications (TSDs) that are $9 \mathrm{bp}$ in length are characteristic of Mutator TIR DNA elements. It has been proposed that Mutator elements might have given rise to MITE derivatives generating $9 \mathrm{bp}$-long TSDs and that 


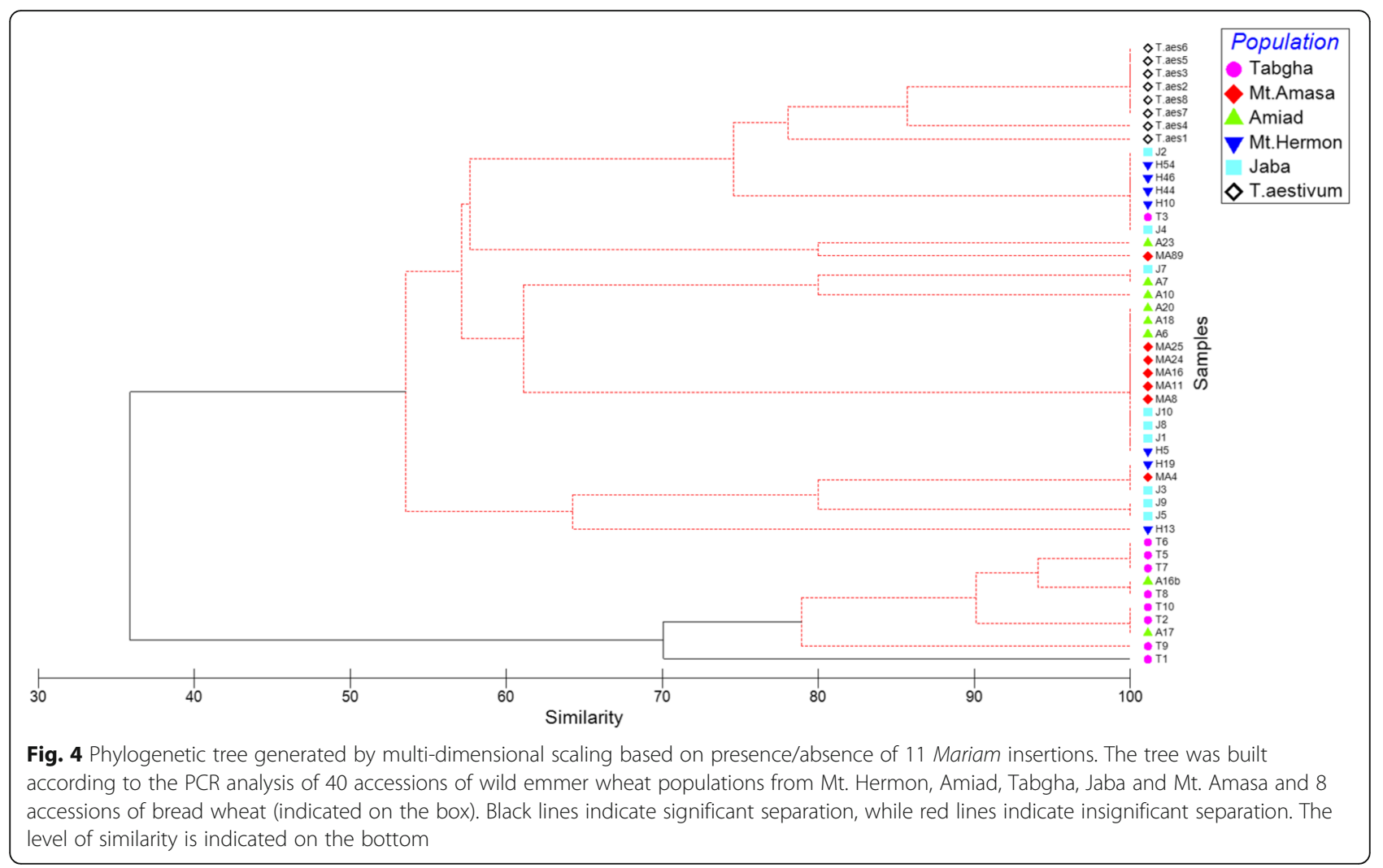

TIRs of some Mutator elements might be very short or even missing [6]. Still, all Mutator-derived MITEs recently annotated and characterized in grasses possess TIRs (see for examples, [34, 35]). Therefore, while Mariam may be a Mutator-derived MITE, it is difficult to classify it based solely on its TSD size.
To further investigate the dynamic nature of Mariam and to examine its insertional patterns in different wheat species, Mariam insertions were retrieved from five publicly available genome drafts of wheat species, and found 33 insertions, some of them were common to wheat species (Additional file 1: Table S1). The bioinformatic

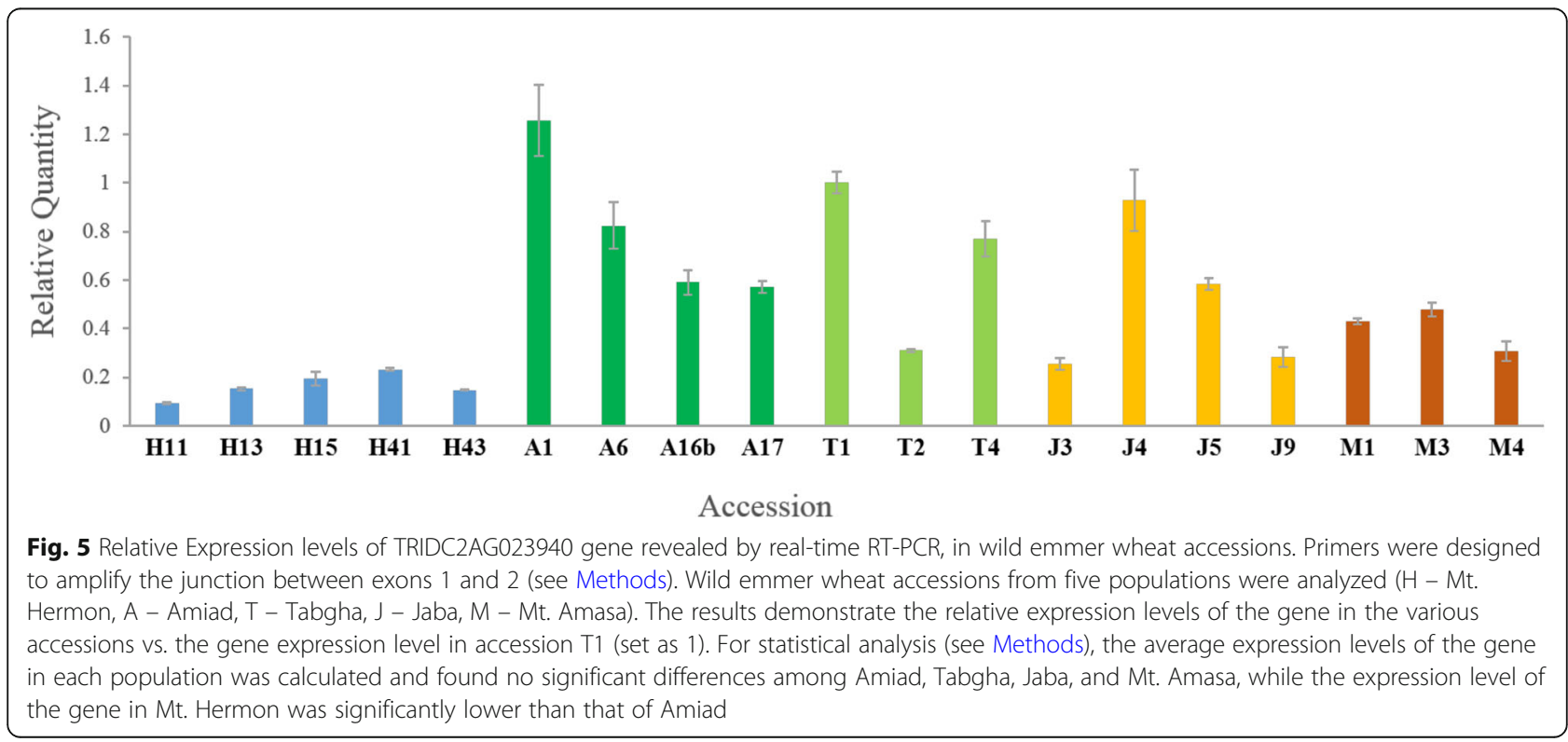




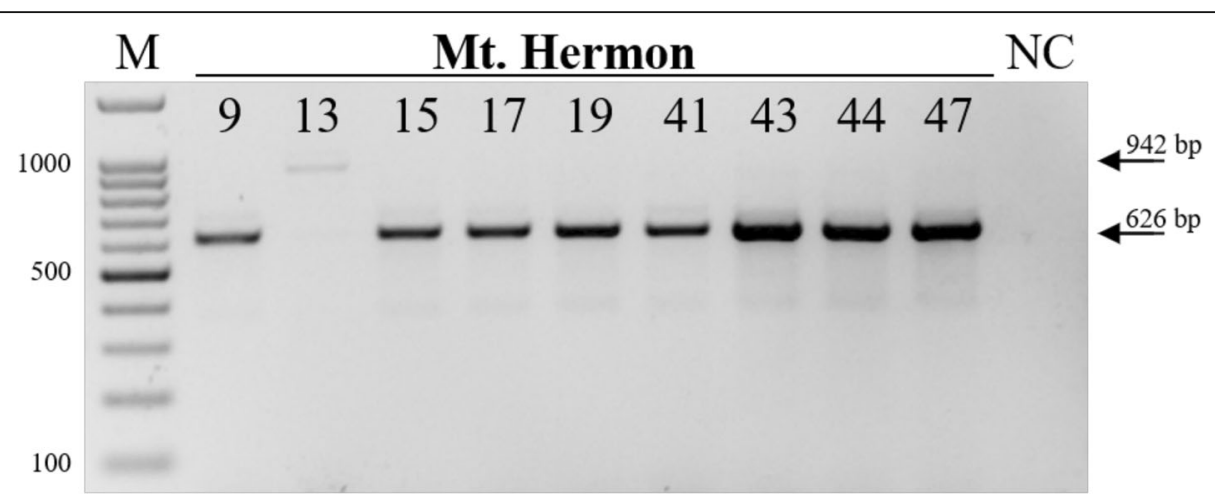

Fig. 6 RT-PCR analysis with primers flanking Mariam in exon 6 of TRIDC2AG023940 gene. The forward primer (AACCGCAATATGCGGATGTT) was complementary to exon 5 (upstream of Mariam insertion), and the reverse primer (TTGTCAATTGTTTGATCAAACAAAGG) was complementary to exon 7 (downstream of Mariam insertion). The RT-PCR reaction was performed in the same conditions as the site-specific PCR analysis in Fig. 1 (see Methods). The upper arrow notes a 942 bp band corresponding with Mariam insertion (in accession 13), while the lower arrow notes a 626 bp band that does not harbor Mariam insertion (in accessions 9, 15-47). M denotes the size marker (the numbers in the left are in bp), while NC denotes a negative control

analyses showed that Mariam is unique to the wheat group and is present in a low copy number in the diploid T. urartu (A genome) and Ae. tauschii (D genome) species, and therefore most likely was also present in the B progenitor of polyploid wheat. The retrieved Mariam insertions of T. turgidum ssp. dicoccoides were validated on accessions collected from 5 different populations and in 8 accessions of bread wheat, and found high polymorphism levels among accessions, for some cases in a population-specific manner (Table 1).

Some of Mariam insertions were found within genic regions, while in most cases Mariam was located in the UTR or inside an intron. A2-MH insertion (the first Mariam insertion discovered in emmer) was found located within exon 6 of a gene coding for 5 -formyltetrahydrofolate cycloligase mitochondrial enzyme. Site-specific PCR analysis showed this insertion was found in only two accessions of Mt. Hermon population of wild emmer wheat, while qRealtime PCR analysis showed decreased expression of this gene in all Mt. Hermon accessions examined, compared to the other wild emmer wheat populations.

\section{Conclusions}

The discovery of a novel mobile element, a sequence that does not possess any internal characteristics of known TEs, indicates the wheat genome probably possesses other mobile sequences that are difficult to detect, with higher copy number and possibly a prominent functional impact. This study emphasizes the huge contribution of updated genome assemblies to wheat studies while revealing potentially interesting genetic variation in wild emmer wheat populations, as well as in different wheat polyploids. Moreover, although the data collected and analyzed to date does not allow the classification of the newly discovered mobile element, it provides additional insight into the diversity of mobile DNA in wheat genomes.

\section{Methods}

Genomic and transcriptomic data

We have used the genome drafts of five Triticum and Aegilops species: (1) T. urartu, the donor of A genome (https://www.ncbi.nlm.nih.gov/assembly/GCA_003

\begin{tabular}{|l} 
a. $\begin{array}{l}\text { Query seq. } \\
\text { Specific hits } \\
\text { Superfanilies }\end{array}$ \\
$\begin{array}{l}\text { b. } \\
\text { Query seq. } \\
\text { Superf anilies }\end{array}$ \\
$\begin{array}{l}\text { Fig. } \mathbf{7} \text { Schematic representation of the conserved protein domains within the coding sequence of TRIDC2AG023940 gene. a protein domains of } \\
\text { the gene that lacks Mariam insertion. } \mathbf{b} \text { protein domains of the gene that harbor Mariam insertion in exon 6. This was carried on by a Blastp } \\
\text { search in the NCBI database. The numbers on top note amino acid positions. Conserved domains or their parts are marked in g reen. Note that } \\
\text { the predicted protein product of the allele harboring Mariam insertion is shorter than the predicted product of the regular allele (249 vs. 280 a.a.) } \\
\text { and lacks part of the predicted cyclo-ligase domain }\end{array}$ \\
\hline
\end{tabular}


073215.1) [36]. (2) Ae. tauschii, the donor of D genome (https://www.ncbi.nlm.nih.gov/assembly/GCA_00034

7335.2) [37]. (3) T. turgidum ssp. diccocoides, wild emmer wheat, genome $A B$ (WEWseq: http://wewseq. wix.com/consortium) [4]. (4) T. aestivum, bread wheat, genome ABD. (https://plants.ensembl.org/Triticum_aestivum/Info/Index) [5, 30]. (5) T. turgidum ssp. durum, durum (pasta) wheat, genome $A B$ [38].

\section{RNA-seq database}

The updated publicly available RNA-seq database of $T$. aestivum and T. turgidum ssp. dicoccoides found in Ensemblplants were used in this study [5, 30]. The library includes cDNA, CDS and ncRNA sequences (https:// plants.ensembl.org/Triticum_aestivum/Info/Annotation/, http://plants.ensembl.org/Triticum_dicoccoides/Info/Annotation/).

\section{Retrieval of Mariam insertions}

We have used the first Mariam sequence identified (A2$\mathrm{MH}$ ) as a query in the MITE analysis kit (MAK) software (http://labs.csb.utoronto.ca/yang/MAK/), [39, 40]. MAK is a homology-based software, meaning it uses a consensus sequence as query and BLASTN algorithm with global alignment. We have used an e-value of $1 \mathrm{e}^{-3}$ and an end mismatch tolerance of 20 nucleotides. In addition, flanking sequences (1000 bp from each end) were retrieved to characterize the insertion sites.

\section{Phylogenetic analysis}

Primer6 software version 6.1.6 [41] was used to construct phylogenetic trees clustering the wild emmer and bread wheat accessions according to the insertional polymorphism of Mariam, based on ss-PCR. Primer6 software performed hierarchical agglomerative clustering analysis of each matrix with Bray-Curtis similarity and used the similarity profile (SIMPROF) test on each node to assess the statistical significance of the phylogenetic trees. SIMPROF calculates a mean profile by randomizing the value of each variable and re-calculating the profile. The pi statistic was calculated as the deviation of the actual resemblance profile of the resemblance matrix from the mean profile. This was compared with the deviation of further randomly generated profiles to test for significance.

\section{Statistical analysis}

Statistica statsoft was used for the analysis of one-way ANOVA. Relative quantity (relative expression of TRIDC2AG023940 gene by q-realtime-PCR) was used as the dependent variable while Population was used as the categorial variable. Analysis of the specific differences between populations was done using Post hoc and Tukey's test.

\section{Plant material and DNA and RNA extraction}

A collection of wild emmer wheat ( $T$. turgidum ssp. dicoccoides) populations from five geographically isolated sites in Israel was used in this study; Mt. Hermon, Amiad, Tabgha, Jaba and Mt. Amasa. The same collection was used in a previous publication [42]. In addition, 8 accessions of $T$. aestivum were used (Additional file 2: Table S2). Seeds were kindly provided by Dr. Sergei Volis (Mt. Hermon, Amiad and Mt. Amasa populations) and by Prof. Eviatar Nevo from the University of Haifa (Tabgha and Jaba populations). Bread wheat accessions were provided by the United States Department of Agriculture (USDA). All wild emmer seeds were provided by Israeli stock centers. Seeds of Tabgha and Jaba Israeli populations are available in the Wild Cereals gene bank (WCGB), Institute of Evolution, University of Haifa. Seeds of Mt. Hermon, Amiad and Mt. Amasa Israeli populations are available in the Lieberman Germplasm Bank, Institute for CerealCrops Improvement, Tel-Aviv University. 10-20 accessions from each population were grown in a greenhouse under common garden conditions. Leaf material was harvested approximately 4 weeks post-germination for DNA extraction using the DNeasy plant mini kit (Qiagen) and for total RNA extraction using TriReagent (Sigma).

\section{Site-specific PCR}

Site-specific PCR primers were designed using Primer3 software (http://bioinfo.ut.ee/primer3-0.4.0/primer3/) to test specific insertion sites. PCR amplifications were prepared with $13.2 \mu \mathrm{l}$ of Ultra-pure water (HyLabs), $2 \mu \mathrm{l}$ of 109 Taq DNA polymerase buffer C (EURx), $0.8 \mu \mathrm{l}$ of 25 $\mathrm{mM} \mathrm{MgCl}_{2}$ (EURx), $0.8 \mu \mathrm{l}$ of $2.5 \mathrm{mM}$ dNTP mix, $0.2 \mu \mathrm{l}$ of Taq DNA polymerase ( $5 \mathrm{U} / \mu \mathrm{l}, \mathrm{EURx}), 1 \mu \mathrm{l}$ of each site-specific primer $(50 \mathrm{ng} / \mu \mathrm{l})$ and $1 \mu \mathrm{l}$ of genomic DNA (50 ng/ $\mu \mathrm{l})$. The conditions for the PCR reactions were as follows: $94^{\circ} \mathrm{C}$ incubation for $3 \mathrm{~min}$, a cycle of $\left(94{ }^{\circ} \mathrm{C}\right.$ for $1 \mathrm{~min}, 57^{\circ} \mathrm{C}$ for $1 \mathrm{~min}, 72^{\circ} \mathrm{C}$ for $1 \mathrm{~min}$ ) repeated 30 times and $72{ }^{\circ} \mathrm{C}$ for $3 \mathrm{~min}$. A $10 \mu \mathrm{l}$ aliquot of the PCR products was tested on a $1.5 \%$ agarose gel and visualized with ethidium bromide (Amresco). The expected product sizes were determined by a DNA size standard (100 bp ladder, SMOBIO).

\section{Single-strand CDNA synthesis}

cDNA for gene expression analysis was synthesized using 5xAll-In-One RT MasterMix (abm) in $20 \mu \mathrm{l}$ reactions. Each reaction contained $4 \mu \mathrm{l}$ of MasterMix and up to $2 \mu \mathrm{g}$ of RNA dissolved in $16 \mu \mathrm{l}$ of Ultra-pure water (HyLabs). The reactions were incubated at $42{ }^{\circ} \mathrm{C}$ for 15 min. The purity of each cDNA sample was tested by PCR using site-specific primers complementary to two exons of the Actin gene, giving different amplification 
products for cDNA and genomic DNA. No genomic DNA contamination was detected.

\section{Gene expression analysis using real-time quantitative RT- PCR}

The expression levels of TRIDC2AG023940 were tested by Real-Time quantitative PCR. Primers specific to the cDNA sequence of this gene were designed with Primer Express v2.0 software. One primer was complementary to the exon-exon junction in order to make the reaction less sensitive to possible DNA contamination of RNA samples. The forward primer was designed to span the junction between exons 1 and 2 to ensure that the reaction would not be sensitive to DNA contamination. Note that no pair of genomespecific primers could be optimized for RT-qPCR. Realtime RT-qPCR analysis was then performed on cDNA samples of 19 accessions from the five populations. qPCR experiments were performed using a 7500 Fast Real-Time PCR system and analyzed using the 7500 version 2.0.5 software (Applied Biosystems). Each reaction contained $7.5 \mu \mathrm{l}$ KAPA SYBR FAST qPCR Master Mix, $0.3 \mu \mathrm{l}$ ROX Low 509 (Kapa Biosystems), $1 \mu \mathrm{l}$ forward and $1 \mu \mathrm{l}$ reverse primers $(10 \mu \mathrm{M}), 0.2 \mu \mathrm{l}$ $\mathrm{dd}_{2} \mathrm{O}$ and $5 \mu$ template cDNA.

Primers efficiency was tested by Real-Time qPCR with each primer pair using serial dilutions of the template cDNA mix to produce a standard curve. The efficiency was calculated by $[(10-1 / y)-1] \times 100 \%$, where $y$ is the standard curve slope. Based on the standard curve, a 50-fold dilution of cDNA was used as template in Real-Time qPCR amplifications for gene expression analysis. To validate product specificity, melting curves were produced and demonstrated a single specific product for each primer pair. Real-Time qPCR analysis was performed as described above using the comparative $2^{-\Delta \Delta C T}$ method, with Actin expression levels serving as an endogenous control and one randomly chosen accession as a reference sample. The relative expression levels of the target gene were assessed using the comparative $2^{-\Delta \Delta C T}$ method, as previously described.

\section{Supplementary information}

Supplementary information accompanies this paper at https://doi.org/10. 1186/s12870-019-2034-1

Additional file 1: Table S1. Intact Mariam insertions retrieved from five wheat species.

Additional file 2: Table S2. T. aestivum accessions used in this study and their geographic origin.

Additional file 3: Table S3. Site-specific primers flanking insertions of Mariam.

Additional file 4: Figure S1. Sequence logo representing target site preference of Mariam. Figure S2. Site-specific PCR analyses of Mariam insertions.

\section{Abbreviations}

MITE: Miniature inverted-repeat transposable elements; TE: Transposable elements; TIR: Terminal inverted repeat; TSD: Target site duplication

\section{Acknowledgements}

We want to thank Dr. Guojun Yang, University of Toronto, for providing the stand-alone version of MAK. We also thank the USDA for providing the bread wheat accessions.

\section{Authors' contributions}

KD carried out in-silico analysis and wet-bench experiments, participated in preparing the original manuscript draft. DKF validated bioinformatics data and PCR analysis, participated in preparing the original manuscript draft. KK carried out the research design, prepared, revised, and submitted the final version of the manuscript. All authors have read and approved the final manuscript.

\section{Funding}

Funded by Israel Science Foundation (grant \# 322/15) https://www.isf.org.il/\#/. Funding was utilized for purchase of materials used in the experiments. The funding agency did not take any role in the experimental design and data analysis.

\section{Availability of data and materials}

All data generated in this study are included in the paper and in the supporting information files.

\section{Ethics approval and consent to participate}

Not applicable.

\section{Consent for publication}

Not applicable.

\section{Competing interests}

The authors declare that they have no competing interests.

\section{Author details}

${ }^{1}$ Present Address: Department of Molecular Biology and Ecology of Plants, Tel-Aviv University, Tel Aviv, Israel. ${ }^{2}$ Department of Life Sciences, Ben-Gurion University, 84105 Beer-Sheva, Israel.

Received: 21 May 2019 Accepted: 12 September 2019 Published online: 01 November 2019

\section{References}

1. Kidwell MG. Transposable elements and the evolution of genome size in eukaryotes. Genetica. 2002;115(1):49-63.

2. Charles M, Belcram H, Just J, Huneau C, Viollet A, Couloux A, Segurens B, Carter M, Huteau V, Coriton O. Dynamics and differential proliferation of transposable elements during the evolution of the $B$ and $A$ genomes of wheat. Genetics. 2008;180(2):1071-86.

3. Clavijo BJ, Venturini L, Schudoma C, Accinelli GG, Kaithakottil G, Wright J, Borrill P, Kettleborough G, Heavens D, Chapman H. An improved assembly and annotation of the allohexaploid wheat genome identifies complete families of agronomic genes and provides genomic evidence for chromosomal translocations. Genome Res. 2017;27(5):885-96.

4. Avni R, Nave M, Barad O, Baruch K, Twardziok SO, Gundlach H, Hale I, Mascher M, Spannagl M, Wiebe K. Wild emmer genome architecture and diversity elucidate wheat evolution and domestication. Science. 2017; 357(6346):93-7.

5. Mayer KF, Rogers J, Doležel J, Pozniak C, Eversole K, Feuillet C, Gill B, Friebe B, Lukaszewski AJ, Sourdille P. A chromosome-based draft sequence of the hexaploid bread wheat (Triticum aestivum) genome. Science. 2014; 345(6194):1251788.

6. Wicker T, Sabot F, Hua-Van A, Bennetzen UL, Capy P, Chalhoub B, Flavell A, Leroy $\mathrm{P}$, Morgante M, Panaud O. A unified classification system for eukaryotic transposable elements. Nat Rev Genet. 2007;8(12):973-82.

7. Lisch D. How important are transposons for plant evolution? Nat Rev Genet. 2012;14(1):49-61.

8. Kumar A, Bennetzen JL. Plant retrotransposons. Annu Rev Genet. 1999;33(1): 479-532. 
9. Capy P, Gasperi G, Biémont C, Bazin C. Stress and transposable elements: co-evolution or useful parasites? Heredity. 2000;85(2):101-6.

10. Soltis DE, Soltis PS. Polyploidy: recurrent formation and genome evolution. Trends Ecol Evol. 1999;14(9):348-52.

11. Casacuberta JM, Santiago N. Plant LTR-retrotransposons and MITEs: control of transposition and impact on the evolution of plant genes and genomes. Gene. 2003;311:1-11.

12. Mansour A. Epigenetic activation of genomic retrotransposons. J Cell Mol Biol. 2007;6(2):99-107.

13. Slotkin RK, Martienssen R. Transposable elements and the epigenetic regulation of the genome. Nat Rev Genet. 2007;8(4):272-85.

14. Zhao D, Ferguson AA, Jiang N. What makes up plant genomes: the vanishing line between transposable elements and genes. Biochim Biophys Acta. 2016;1859(2):366-80.

15. Rebollo R, Romanish MT, Mager DL. Transposable elements: an abundant and natural source of regulatory sequences for host genes. Annu Rev Genet. 2012;46:21-42.

16. Wessler SR. Eukaryotic transposable elements: teaching old genomes new tricks. Implicit Genome. 2006. pp. 138-65.

17. Schmitz J, Brosius J. Exonization of transposed elements: a challenge and opportunity for evolution. Biochimie. 2011;93(11):1928-34.

18. Keidar D, Doron C, Kashkush K. Genome-wide analysis of a recently active retrotransposon, $\mathrm{Au}$ SINE, in wheat: content, distribution within subgenomes and chromosomes, and gene associations. Plant Cell Rep. 2018;37(2):193-208.

19. Hawkins JS, Proulx SR, Rapp RA, Wendel JF. Rapid DNA loss as a counterbalance to genome expansion through retrotransposon proliferation in plants. Proc Natl Acad Sci. 2009;106(42):17811-6.

20. Feldman M, Levy A. Allopolyploidy-a shaping force in the evolution of wheat genomes. Cytogenet Genome Res. 2005;109(1-3):250-8.

21. Petersen G, Seberg O, Yde M, Berthelsen K. Phylogenetic relationships of Triticum and Aegilops and evidence for the origin of the A, B, and D genomes of common wheat (Triticum aestivum). Mol Phylogenet Evol. 2006;39(1):70-82.

22. Aaronsohn A. The discovery of wild wheat. City Club Bull Chicago. 1913;6: 167-73.

23. Moseman JG, Nevo E, Morshidy ME, Zohary D. Resistance of Triticum dicoccoides to infection with Erysiphe graminis tritici. Euphytica. 1984;33(1):41-7.

24. Nevo E, Krugman T, Beiles A. Genetic resources for salt tolerance in the wild progenitors of wheat (Triticum dicoccoides) and barley (Hordeum spontaneum) in Israel. Plant Breed. 1993;110(4):338-41.

25. Krugman T, Korol A, Nevo E, Snape JW, Levy O, Rubin B. Comparative RFLP mapping of the chlorotoluron resistance gene (Su1) in cultivated wheat (Triticum aestivum) and wild wheat (Triticum dicoccoides). Theor Appl Genet. 1997;94(1):46-51.

26. Krugman T, Chagué V, Peleg Z, Balzergue S, Just J, Korol AB, Nevo E, Saranga $Y$, Chalhoub B, Fahima T. Multilevel regulation and signalling processes associated with adaptation to terminal drought in wild emmer wheat. Funct Integr Genomics. 2010;10(2):167-86.

27. Nevo E, Beiles A. Genetic diversity of wild emmer wheat in Israel and Turkey: structure, evolution, and application in breeding. Theor Appl Genet. 1989;77(3): 421-55.

28. Volis S, Song M, Zhang Y-H, Shulgina I. Fine-scale spatial genetic structure in emmer wheat and the role of population range position. Evol Biol. 2014; 41(1):166-73.

29. Domb K, Keidar D, Yaakov B, Khasdan V, Kashkush K. Transposable elements generate population-specific insertional patterns and allelic variation in genes of wild emmer wheat (Triticum turgidum ssp. dicoccoides). BMC Plant Biol. 2017;17(1):175.

30. Appels R, Eversole K, Feuillet C, Keller B, Rogers J, Stein N, Pozniak CJ, Choulet F, Distelfeld A, Poland J. Shifting the limits in wheat research and breeding using a fully annotated reference genome. Science. 2018; 361(6403):eaar7191.

31. Wicker T, Matthews DE, Keller B. TREP: a database for Triticeae repetitive elements. Trends Plant Sci. 2002;7(12):561-2.

32. Bao W, Kojima KK, Kohany O. Repbase update, a database of repetitive elements in eukaryotic genomes. Mob DNA. 2015;6(1):11.

33. Crooks GE, Hon G, Chandonia J-M, Brenner SE. WebLogo: a sequence logo generator. Genome Res. 2004;14(6):1188-90.

34. Yang G, Hall TC. MDM-1 and MDM-2: two mutator-derived MITE families in rice. J Mol Evol. 2003;56(3):255-64.
35. Mo Y-J, Kim K-Y, Shin W-C, Lee G-M, Ko J-C, Nam J-K, Kim B-K, Ko J-K, Yu Y, Yang T-J. Characterization of Imcrop, a Mutator-like MITE family in the rice genome. Genes Genomics. 2012;34(2):189-98.

36. Ling $H-Q$, Ma B, Shi $X$, Liu H, Dong L, Sun H, Cao Y, Gao Q, Zheng S, Li Y. Genome sequence of the progenitor of wheat A subgenome Triticum urartu. Nature. 2018:557(7705):424.

37. Luo M-C, Gu YQ, Puiu D, Wang H, Twardziok SO, Deal KR, Huo N, Zhu T, Wang $L$, Wang $Y$. Genome sequence of the progenitor of the wheat $D$ genome Aegilops tauschii. Nature. 2017;551:498-502.

38. Maccaferri M, Harris NS, Twardziok SO, Pasam RK, Gundlach H, Spannagl M, Ormanbekova D, Lux T, Prade VM, Milner SG. Durum wheat genome highlights past domestication signatures and future improvement targets. Nat Genet. 2019;51:885-95.

39. Janicki M, Rooke R, Yang G. Bioinformatics and genomic analysis of transposable elements in eukaryotic genomes. Chromosom Res. 2011;19(6): 787-808.

40. Yang G, Hall TC. MAK, a computational tool kit for automated MITE analysis. Nucleic Acids Res. 2003;31(13):3659-65.

41. Clarke KR. Non-parametric multivariate analyses of changes in community structure. Aust J Ecol. 1993;18(1):117-43.

42. Venetsky A, Levy-Zamir A, Khasdan V, Domb K, Kashkush K. Structure and extent of DNA methylation-based epigenetic variation in wild emmer wheat (T. turgidum ssp. dicoccoides) populations. BMC Plant Biol. 2015;15:200.

\section{Publisher's Note}

Springer Nature remains neutral with regard to jurisdictional claims in published maps and institutional affiliations.

Ready to submit your research? Choose BMC and benefit from:

- fast, convenient online submission

- thorough peer review by experienced researchers in your field

- rapid publication on acceptance

- support for research data, including large and complex data types

- gold Open Access which fosters wider collaboration and increased citations

- maximum visibility for your research: over $100 \mathrm{M}$ website views per year

At $\mathrm{BMC}$, research is always in progress.

Learn more biomedcentral.com/submissions 